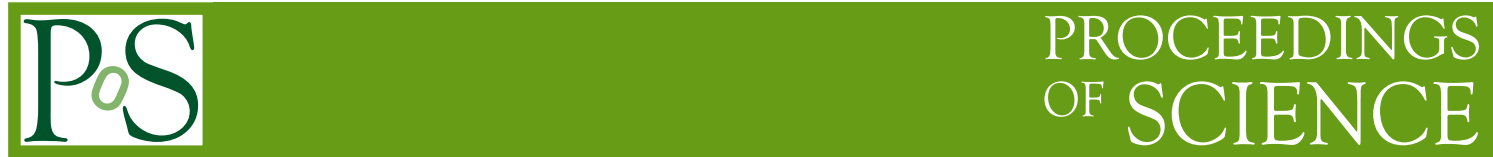

\title{
Phase of the Fermion Determinant for QCD at Finite Chemical Potential
}

\section{K. Splittorff}

Niels Bohr Institute, Blegdamsvej 17, Copenhagen, Denmark

E-mail: split@nbi.dk

\section{J.J.M. Verbaarschot}

Stony Brook University, Stony Brook, NY 11794

E-mail: jacobus.verbaarschotestonybrook.edu

In this lecture we discuss various properties of the phase factor of the fermion determinant for QCD at nonzero chemical potential. Its effect on physical observables is elucidated by comparing the phase diagram of QCD and phase quenched QCD and by illustrating the failure of the Banks-Casher formula with the example of one-dimensional QCD. The average phase factor and the distribution of the phase are calculated to one-loop order in chiral perturbation theory. In quantitative agreement with lattice QCD results, we find that the distribution is Gaussian with a width $\sim \mu T \sqrt{V}$ (for $m_{\pi} \ll T \ll \Lambda_{\mathrm{QCD}}$ ). Finally, we introduce, so-called teflon plated observables which can be calculated accurately by Monte Carlo even though the sign problem is severe. 


\section{Introduction}

Although the Euclidean formulation of QCD has been extremely successful in the evaluation of observables from first principles, at nonzero chemical potential, because of the phase of the fermion determinant, progress has been slow and limited to a small part of the QCD phase diagram (see for example [1] for a recent review). Motivated by the drastic effect of the phase of fermion determinant on physical observables, we study its properties to one-loop order in chiral perturbation theory. Since the average phase factor is a ratio of two partition functions it has to vanish exponentially with the volume when evaluated with respect to a positive definite measure. Therefore, a sensible strategy is to extract observables by extrapolation from small volumes. In the domain of validity of chiral perturbation theory it possible to derive the volume dependence of the average phase factor, and to determine the parameter range where reweighting methods $[2,3]$ work. The distribution of the phase, which is also calculated, might be relevant in applications of the density of states method $[4,5,6]$. It is less clear how methods that rely on analytical continuation in the chemical potential $[7,8,9,10]$ or methods that are based on the canonical partition function $[11,12]$ are affected by the sign problem, but each method has its own difficulties.

With regards to the volume dependence of the QCD partition function at $\mu \neq 0$, this can be investigated in detail in the microscopic domain of QCD $[13,14]$ where QCD is equivalent to chiral random matrix theory. In this domain, the cancellations that give rise to the discontinuity [15] of the mass dependence of the chiral condensate also take place for finite size matrices [16].

After the definition of the average phase factor in section 2 , we discuss its effects on the phase diagram and on the chiral condensate in 1d QCD in Section 3. Results obtained with chiral perturbation theory including the distribution of the phase are given in sections 4 and 5 .

\section{Average Phase Factor}

At nonzero chemical potential, $\mu$, the QCD Dirac operator is given by $D+\mu \gamma_{0}+m$, where $D$ is the anti-hermitian Dirac operator at zero chemical potential, and $m$ is the quark mass. Because

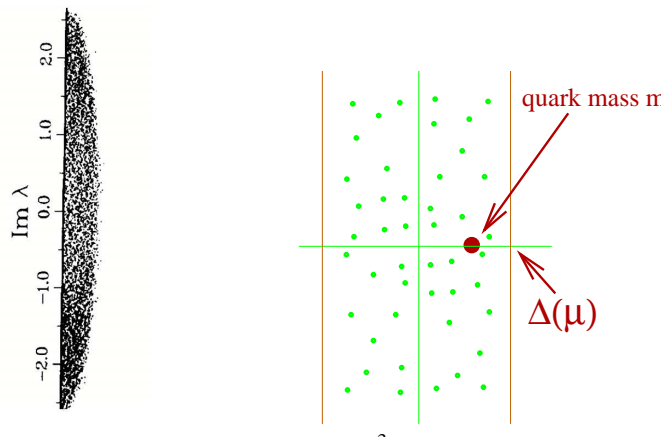

Fig. 1. Quenched Dirac eigenvalues on a $4^{3} \times 8$ lattice [17] (left) and a schematic Dirac spectrum (right). $\mu \gamma_{0}$ is hermitian, this Dirac operator does not have any hermiticity properties and its eigenvalues are scattered in the complex plane (See Fig. 1). Although the Dirac eigenvalues are still paired, the determinant is complex $\operatorname{det}\left(D+\mu \gamma_{0}+m\right)=e^{i \theta}\left|\operatorname{det}\left(D+\mu \gamma_{0}+m\right)\right|$. The phase will be strongly fluctuating if the quark mass is inside the domain of eigenvalues resulting in an average phase factor that vanishes in the thermodynamic limit.

The average can be evaluated with respect to different partition functions. A particularly useful definition of the average is with respect to the phase quenched partition function,

$$
\left\langle e^{2 i \theta}\right\rangle=\frac{\left\langle\left(\operatorname{det}\left(D+\mu \gamma_{0}+m\right)\right)^{2}\right\rangle}{\left\langle\left|\operatorname{det}\left(D+\mu \gamma_{0}+m\right)\right|^{2}\right\rangle}=\frac{Z_{N_{f}=2}^{\mathrm{QCD}}}{Z_{N_{f}=2}^{|\mathrm{QCD}|}},
$$


which is the ratio of the QCD partition function and the phase quenched QCD partition function. Because free energies are extensive and the free energy of QCD and phase quenched QCD is generally different, the average phase factor vanishes exponentially with the volume. This explains the severity of the sign problem.

In addition to the sign problem, there is an overlap problem. For any given field configuration, the chiral condensate is close to the result for the quenched or phase quenched partition function. The true value of the condensate is due to exceptional gauge field configurations. The deep reason for this is that determinants and the average phase factor depend exponentially on the volume.

\section{The effect of the phase factor}

The simplest way to analyze the effect of the phase factor is to compare the phase diagram of QCD and phase quenched QCD (denoted by IQCDI). First principle calculations for QCD have only been done at or near the temperature axis of the phase diagram in the $\mu T$-plane. At zero temperature, a transition to a phase with nonzero baryon density takes place at $\mu \approx m_{N} / 3$. Experimentally [18], it has been shown that this transition is first order with the first order line ending in a critical endpoint (see Fig. 2). The remainder of the phase diagram is based on general arguments and model studies. At extremely high densities, one expects a superconducting phase whereas at intermediate densities a confined phase with restored chiral symmetry is a likely possibility $[19,20]$.
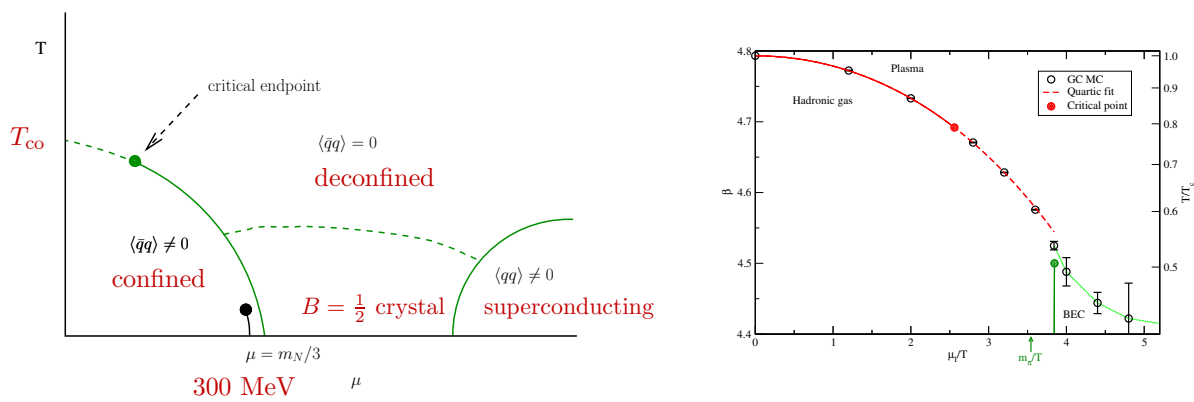

Fig. 2. The phase diagram of QCD in the temperature baryon chemical potential plane (left) and in the temperature isospin chemical potential plane (right). The data points in the right figure are from lattice QCD simulations [21].

On inspection of the lattice Dirac spectra at nonzero chemical potential (see Fig. 1) we notice that the eigenvalues are distributed more or less homogeneously inside a strip and that the strip has a sharp edge. This implies that we can expect a phase transition when the quark mass hits the edge of this strip located at $\Delta(\mu)$. The critical chemical potential is therefore given by $\Delta\left(\mu_{c}\right)=m$ $[22,23]$. Because phase quenched QCD is QCD at nonzero isospin chemical potential, we know that at low temperatures a phase transition to a pion condensed phase occurs at $\mu=m_{\pi} / 2$. Using the Gell-Mann-Oakes-Renner relation this results in a half-width of the strip of $\Delta(\mu)=2 \mu^{2} F^{2} / \Sigma$.

The width Dirac spectrum for each gauge field configuration in full QCD is also given by $2 \Delta(\mu)$, but instead of a phase transition at $\mu=m_{\pi} / 2$ there is a phase transition at $\mu=m_{N} / 3$. In particular, this implies that the chiral condensate should be a smooth function of the chemical potential in the region with zero baryon density, and in the low temperature limit, it should not 
depend at all on the chemical potential. How this can happen in spite of the fact that the Dirac spectrum is $\mu$-dependent has been understood in detail for the microscopic domain of QCD [15, 16]. Here we will illustrate this phenomenon for QCD in one-dimension [24].

\subsection{Effect of the Phase in 1d QCD}

The Banks-Casher relation [25] states that

$$
\Sigma=\lim _{m \rightarrow 0} \lim _{V \rightarrow \infty} \frac{\pi \rho(m)}{V}
$$

where $\rho(\lambda)$ is the density of Dirac eigenvalues on the imaginary axis and $V$ is the volume of spacetime. Although originally intended for an anti-Hermitian Dirac operator, this relation correctly gives a vanishing chiral condensate for phase quenched QCD at $\mu \neq 0$ since there is no accumulation of eigenvalues on the imaginary axis. However, for full QCD at $\mu \neq 0$, the chiral condensate has a discontinuity when the quark mass crosses the imaginary axis, but it does so without an accumulation of eigenvalues on the imaginary axis. The alternative mechanism that is at work was first discovered in random matrix theory $[15,16]$. Below we will illustrate this mechanism for QCD in one dimension which can also be viewed as a random matrix model.
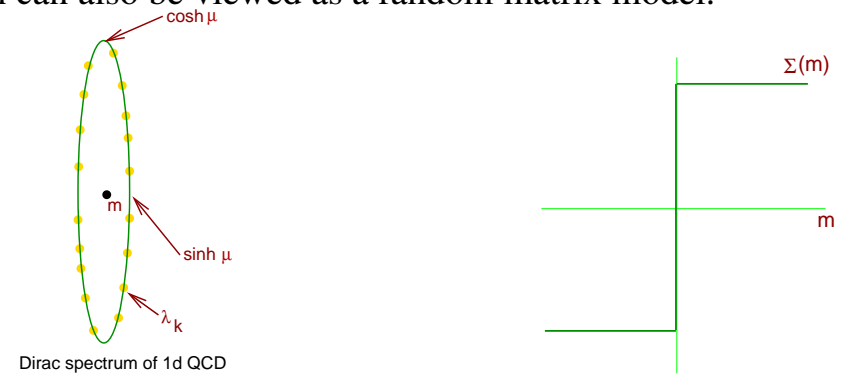

Fig. 3. Schematic plot of eigenvalues of the Dirac operator for lattice QCD in one dimension (left). The yellow dots denote the position of the eigenvalues for a single gauge field configuration, whereas the green ellipse shows the support of the spectrum in the thermodynamic limit. The chiral condensate for one flavor versus the quark mass is shown in the right figure.

The partition function of lattice QCD in one dimension is given by

$$
Z=\int_{U \in U\left(N_{c}\right)} d U \operatorname{det} D
$$

where the integral is over the Haar-measure of $U\left(N_{c}\right)$. The 1d lattice Dirac operator is the $N \times N_{c}$ matrix with hopping matrix elements given by $U \exp (\mu)$ and its inverse [26] (the number of lattice points is denoted by $N$ ). The chiral condensate for one flavor is given by

$$
\Sigma(m)=\frac{\left\langle\frac{1}{N} \sum_{k} \frac{1}{\lambda_{k}+m} \prod_{k}\left(\lambda_{k}+m\right)\right\rangle}{\left\langle\prod_{k}\left(\lambda_{k}+m\right)\right\rangle} .
$$

Since the eigenvalues, $\lambda_{k}$, are located on an ellipse in the complex plane (see Fig. 3) the determinant has a complex phase. For $U(1)$ the chiral condensate can be evaluated analytically [24] with the result that is shown in the right figure of Fig. 3. The amazing phenomenon, also known as the "Silver Blaze Problem" [27], is that the chiral condensate is continuous when $m$ crosses the ellipse of eigenvalues, but shows a discontinuity at $m=0$, where there are no eigenvalues. This can happen because the chiral condensate is determined by exponentially large (in the number of lattice points) contributions which cancel to give a finite result for $N \rightarrow \infty$. 


\section{Average Phase Factor in Chiral Perturbation Theory}

Because the average phase factor is the ratio of the QCD partition function and the phase quenched QCD partition function, it can be evaluated by means of chiral perturbation theory. The low-temperature limit of QCD is given by a gas of pions and does not depend on $\mu$ to one-loop order. Since the phase quenched partition function is QCD at non zero isospin chemical potential the pions are charged with respect to $\mu$ resulting in a $\mu$-dependent one-loop result. For $N_{f}=2$ there are two charged Goldstone modes $\pi_{ \pm}$. Denoting their one-loop contribution by

$\prod_{p} \frac{1}{\sqrt{m_{\pi}^{2}+\vec{p}^{2}+\left(p_{0}-2 i \mu\right)^{2}}} \equiv e^{G_{0}(\mu) / 2}$

we obtain (the product is over all pions)

$$
\begin{aligned}
\left\langle\operatorname{det}^{2}\left(D+m+\mu \gamma_{0}\right)\right\rangle & \sim e^{-V F^{(0)}} \prod_{k} e^{G_{0}(\mu=0) / 2}, \\
\left\langle\left|\operatorname{det}\left(D+m+\mu \gamma_{0}\right)\right|^{2}\right\rangle & \sim e^{-V F^{(0)}} \prod_{k} e^{G_{0}(\mu) / 2} .
\end{aligned}
$$

The average phase factor is the ratio of the two partition functions cf. (2.1) resulting in the cancellation of the neutral Goldstone bosons so that

$$
\left\langle e^{2 i \theta}\right\rangle_{\mathrm{pq}}=e^{G_{0}(\mu=0)-G_{0}(\mu)} .
$$
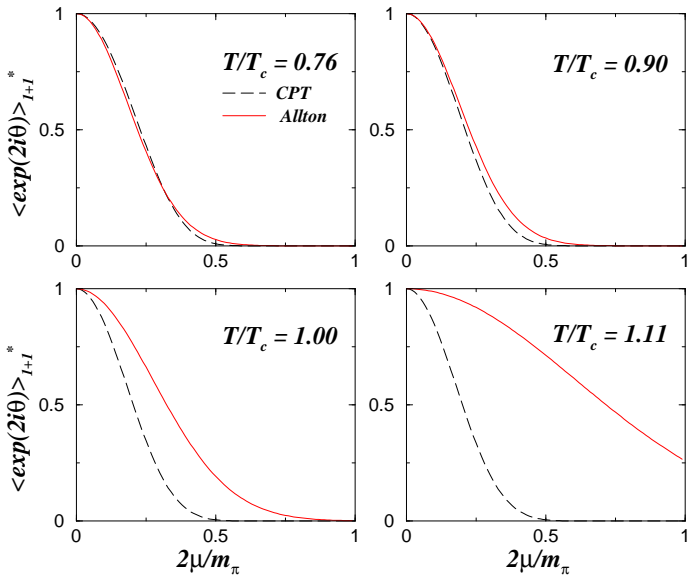

Fig. 4. Average phase factor in lattice QCD obtained from [7] (red curve) compared to one loop chiral perturbation theory in a box equal to the size of the lattice (dashed curve) .

In Fig. 4 where we compare one loop results for the average phase factor to lattice QCD results obtained by Taylor expansion in the chemical potential (there are no fitting parameters). The agreement is striking even for $T / T_{c}=0.90$. As expected chiral perturbation theory fails above $T_{c}$.

\section{Probability Distribution of the Phase}

The density of the phase angle is defined by $\rho(\phi)=\left\langle\delta\left(\phi-\operatorname{Im} \log \operatorname{det}\left(D+m+\mu \gamma_{0}\right)\right)\right\rangle_{N_{f}}$. Notice that $\phi \in\langle-\infty, \infty\rangle$. If the average is over dynamical quarks, the phase density factorizes into an overall phase factor and the phase density of the phase quenched partition function $\left(\rho_{\mathrm{pq}}(\phi)\right)$,

$$
\left\langle\delta(\phi-\theta) e^{i N_{f} \theta}\left|\operatorname{det}^{N_{f}}\left(D+m+\mu \gamma_{0}\right)\right|\right\rangle=e^{i N_{f} \phi}\left\langle\delta(\phi-\theta)\left|\operatorname{det}^{N_{f}}\left(D+m+\mu \gamma_{0}\right)\right|\right\rangle .
$$

According to the Central Limit Theorem we expect that $\rho_{\mathrm{pq}}(\phi)$ approaches a Gaussian distribution [28]. Notice however that observables are determined by correlations with the phase of the fermion determinant, and knowing the Gaussian distribution is only part of the story.

The phase density can be evaluated using the replica trick and chiral perturbation theory [29]. Fourier transforming the $\delta$-function we obtain

$$
\rho_{N_{f}}(\phi)=\left\langle\delta\left(\phi-\operatorname{Im} \log \operatorname{det}\left(D+m+\mu \gamma_{0}\right)\right)\right\rangle_{N_{f}}=\left\langle\sum_{n} e^{i n\left(\phi-\operatorname{Im} \log \operatorname{det}\left(D+m+\mu \gamma_{0}\right)\right.}\right\rangle_{N_{f}}
$$

The phase density therefore follows from the moments of the phase factor,

$$
\left\langle e^{2 i n \theta}\right\rangle_{N_{f}}=\frac{1}{Z_{N_{f}}}\left\langle\frac{\operatorname{det}^{n+N_{f}}\left(D+m+\mu \gamma_{0}\right)}{\operatorname{det}^{n}\left(D^{\dagger}+m+\mu \gamma_{0}\right)}\right\rangle,
$$


which is the ratio of two partition functions with a numerator that contains both bosonic and fermionic Goldstone particles. All $2 n\left(n+N_{f}\right)$ charged Goldstone particles are fermions while the uncharged Goldstone particles are bosons. We thus find

$$
\left\langle e^{2 i n \theta}\right\rangle_{N_{f}}=e^{n\left(n+N_{f}\right)\left[G_{0}(\mu=0)-G_{0}(\mu)\right]} .
$$

By Poisson resummation we obtain $\left(\Delta G=G_{0}(\mu)-G_{0}(\mu=0)\right)$

$\rho(\phi)=\sum_{n} e^{i n \phi} e^{-n / 2\left(n / 2+N_{f}\right) \Delta G}=\frac{e^{\frac{1}{4} N_{f}^{2} \Delta G}}{\sqrt{\pi \Delta G}} e^{i N_{f} \phi-\frac{\phi^{2}}{\Delta G}}$.

A simple expression for the width of the Gaussian is obtained in a small $\mu$ expansion of the ChPT result. For the extensive quantity $\Delta G$ we find $\left(m_{\pi} \ll T\right)$

$$
\Delta G \sim V \mu^{2} T^{2} .
$$

Since the width of the Gaussian $\sim \sqrt{\Delta G}$ this result is in quantitative agreement with the lattice results by Ejiri [28] (See Fig. 5).

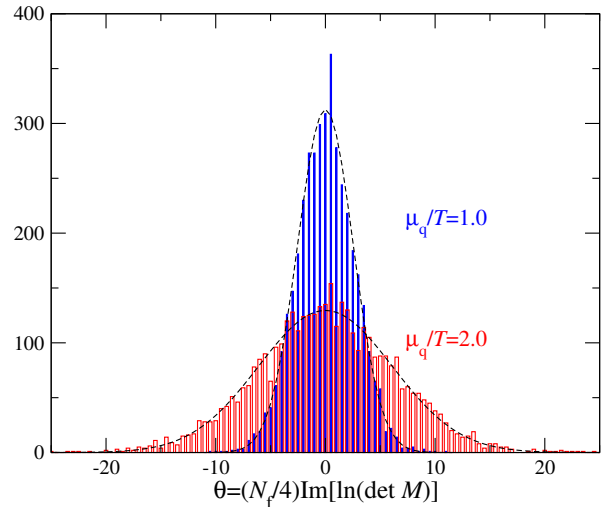

Fig. 5. Phase density in lattice QCD [28].

The average phase factor can also be evaluated in a weak coupling expansion valid at high temperatures [30, 31]. The small- $\mu$ limit of the first nonvanishing contribution is given by [29]

$$
F_{\mathrm{QCD}}(\mu, T)-F_{|\mathrm{QCD}|}(\mu, T) \sim V \alpha_{s}^{2} \mu^{2} T^{2},
$$

with $V$ the space-time volume. This implies that the sign problem remains severe in the thermodynamic limit of the weak coupling result. Notice that expectation values can be calculated accurately despite the sign problem if the correlations between the observable and the phase factor are small. Such teflon plated observables should obey the condition (here $N_{f}=2$ )

$$
\frac{\left|\left\langle\mathscr{O} e^{2 i \theta}\right\rangle_{|\mathrm{QCD}|}-\langle\mathscr{O}\rangle_{|\mathrm{QCD}|}\left\langle e^{2 i \theta}\right\rangle_{|\mathrm{QCD}|}\right|}{\langle\mathscr{O}\rangle_{|\mathrm{QCD}|}\left\langle e^{2 i \theta}\right\rangle_{|\mathrm{QCD}|}} \ll 1
$$

This condition only makes sense if $\langle\mathscr{O}\rangle_{|\mathrm{QCD}|} \neq 0$ as can be seen by rewriting the 1.h.s. as

$$
\frac{\left|\langle\mathscr{O}\rangle_{\mathrm{QCD}}-\langle\mathscr{O}\rangle_{|\mathrm{QCD}|}\right|}{\langle\mathscr{O}\rangle_{|\mathrm{QCD}|}}
$$

For example, when $\mathscr{O}$ is the baryon density this ratio is $O\left(\alpha_{s}^{2}\right)$ whereas $\langle\mathscr{O}\rangle_{\mathrm{QCD}}$ is $O\left(\alpha_{s}^{0}\right)$ [32].

\section{Conclusions}

The phase factor of the fermion determinant completely alters the properties and the physics of the QCD partition function at nonzero chemical potential. We have illustrated this by the relation between the chiral condensate and the Dirac spectrum and the difference between the phase diagram of QCD and phase quenched QCD. Using one-loop chiral perturbation theory, we have determined both the magnitude of the average phase factor and the statistical distribution of the phase which turns out be a Gaussian with width $\sim \mu T \sqrt{V}$. Finally, we introduced a class of observables (teflon plated observables) that can be evaluated accurately despite the sign problem. An example of such observable is the baryon density at high temperature and small chemical potential.

Acknowledgments: This work was supported in part by U.S. DOE Grant No. DE-FAG-88ER40388. 


\section{References}

[1] O. Philipsen, Eur. Phys. J. ST 152, 29 (2007) [arXiv:0708.1293 [hep-lat]].

[2] I. M. Barbour, S. E. Morrison, E. G. Klepfish, J. B. Kogut and M. P. Lombardo, Nucl. Phys. Proc. Suppl. 60A, 220 (1998) [arXiv:hep-lat/9705042].

[3] Z. Fodor and S. D. Katz, JHEP 0404, 050 (2004) [arXiv:hep-lat/0402006].

[4] V. Azcoiti, G. Di Carlo, A. Galante and V. Laliena, JHEP 0412, 010 (2004) [arXiv:hep-lat/0409157].

[5] J. Ambjorn, K. N. Anagnostopoulos, J. Nishimura and J. J. M. Verbaarschot, JHEP 0210, 062 (2002) [arXiv:hep-lat/0208025].

[6] Z. Fodor, S. D. Katz and C. Schmidt, JHEP 0703, 121 (2007) [arXiv:hep-lat/0701022].

[7] C. R. Allton et al., Phys. Rev. D 66, 074507 (2002) [arXiv:hep-lat/0204010].

[8] R. V. Gavai, S. Gupta and P. Majumdar, Phys. Rev. D 65, 054506 (2002) [arXiv:hep-lat/0110032].

[9] M. D'Elia and M. P. Lombardo, Phys. Rev. D 67, 014505 (2003) [arXiv:hep-lat/0209146].

[10] P. de Forcrand and O. Philipsen, Nucl. Phys. B 642, 290 (2002) [arXiv:hep-lat/0205016].

[11] K. F. Liu, arXiv:hep-lat/0312027.

[12] S. Ejiri, arXiv:0804.3227 [hep-lat];

[13] J. J. M. Verbaarschot, Phys. Rev. Lett. 72, 2531 (1994) [arXiv:hep-th/9401059].

[14] E. V. Shuryak and J. J. M. Verbaarschot, Nucl. Phys. A 560, 306 (1993) [arXiv:hep-th/9212088].

[15] J. C. Osborn, K. Splittorff and J. J. M. Verbaarschot, Phys. Rev. Lett. 94, 202001 (2005), [arXiv:hep-th/0501210].

[16] J. C. Osborn, K. Splittorff and J. J. M. Verbaarschot, arXiv:0805.1303 [hep-th].

[17] I. Barbour, N. E. Behilil, E. Dagotto, F. Karsch, A. Moreo, M. Stone and H. W. Wyld, Nucl. Phys. B 275, 296 (1986).

[18] HEMCGC and HTMCGC collaborations, Nucl. Phys. B (Proc. Suppl.) 30 (1993) 315.

[19] L. Castillejo, P. S. J. Jones, A. D. Jackson, J. J. M. Verbaarschot and A. Jackson, Nucl. Phys. A 501, 801 (1989).

[20] L. McLerran and R. D. Pisarski, Nucl. Phys. A 796, 83 (2007) [arXiv:0706.2191 [hep-ph]].

[21] P. dE Forcrand, M. A. Stephanov and U. Wenger, PoS LAT2007, 237 (2007) [arXiv:0711.0023].

[22] P. E. Gibbs, Glasgow preprint 86-0389 (unpublished).

[23] D. Toublan and J. J. M. Verbaarschot, Int. J. Mod. Phys. B 15, 1404 (2001) [hep-th/0001110].

[24] L. Ravagli and J. J. M. Verbaarschot, Phys. Rev. D 76, 054506 (2007) [arXiv:0704.1111 [hep-th]].

[25] T. Banks and A. Casher, Nucl. Phys. B169, 103 (1980).

[26] N. Bilic and K. Demeterfi, Phys. Lett. B 212, 83 (1988).

[27] T. D. Cohen, Phys. Rev. Lett. 91, 222001 (2003) [arXiv:hep-ph/0307089].

[28] S. Ejiri, Phys. Rev. D 77, 014508 (2008) [arXiv:0706.3549 [hep-lat]].

[29] K. Splittorff and J. J. M. Verbaarschot, Phys. Rev. D 77, 014514 (2008) [arXiv:0709.2218 [hep-lat]].

[30] A. Vuorinen, Phys. Rev. D 68, 054017 (2003) [arXiv:hep-ph/0305183].

[31] A. Ipp and A. Rebhan, JHEP 0306, 032 (2003) [arXiv:hep-ph/0305030].

[32] M.-P. Lombarbo, Talk at INT Seattle, August 2008. 